\title{
Die intermediale Differenz-Form-des-Dazwischen im (spät)romantischen Lied am Beispiel von Traum durch die Dämmerung - Vítězslav Nováks „musikalische Polemik"
}

The Intermedia Difference-Form-of-the-in-Between in the (Post) Romantic Song on the Example of Traum durch die Dämmerung „Musical Polemics" of Vítězslav Novák

Petr Pytlík / pytlik@ped.muni.cz

Department of German language and literature, Faculty of Education, Masaryk University, Brno, CZ

\begin{abstract}
The present article examines the fundamentals of musical polemics such as the Czech post-romantic composer Vítězslav Novák conducted against Richard Strauss. Based on the foundations of modern theories of intermediality (Zemanek 2012, Wirth 2004) and (post) romantic intermedial aesthetics (Schmidt 2005), a comparative formalistic and structural analysis of the musical version of Otto Julius Bierbaum's poem Traum durch die Dämmerung by Strauss and Novák is carried out. Using this song as an example, it is examined on which structural levels the musical polemics in Nováks composition are realized, to what extent they represent a specific composition technique and whether and how they call into question the omnipresent idea of romantic "absolute music" (Dahlhaus 1978).
\end{abstract}

\section{Key words}

Vítězslav Novák, Otto Julius Bierbaum, Richard Strauss, intermediality, aesthetics of (post)romantism, music and literature, interart studies 
Anders als in der deutschen romantischen Tradition finden wir in der tschechischen Musik der Romantik und Neuromantik weniger Vertonungen artifizieller Poesie. Folglich gibt es auch in den tschechischen Kunstwissenschaften relativ wenige Studien zum Thema der intermedialen Symbiose von Gedichten und Musik. Im Unterschied dazu kann der deutsche Kunstwissenschaftler Wolf Gerhard Schmidt behaupten, dass die Zahl der Beiträge in der deutschen Literatur- und Musikwissenschaft, die das Beziehungsnetz zwischen beiden Systemen analysieren, kaum zu übersehen sei. Zugleich stellt er aber fest, dass die theoretischen Grundlagen dieser Analysen oft auf Forschungen basieren, die intermediale Bezüge in unterschiedlichen Kunstbereichen beschreiben und für konkrete Analysen des Beziehungsnetzes zwischen Literatur und Musik nur im eingeschränkten Maße beitragen können. ${ }^{1}$ Aufgrund dieses Standpunktes kritisiert Schmidt die eingeführten Intermedialitätsmodelle, wie beispielsweise jene von Rajewsky, die er als filmzentriert bezeichnet, und stellt fest, dass sie methodisch kaum Substantielles für den Bereich Schriftlichkeit/Musikalisierung bieten. ${ }^{2}$ Schmidt selbst übernimmt die Typologie von Steven Paul Scher, der drei Aspekte intermedialer Referenzialität zwischen der Literatur und Musik unterscheidet: 1) Literatur in der Musik (Programmmusik), 2) Musik und Literatur (Vokalmusik), 3. Musik in der Literatur (musikalische Strukturadaption, sprachliche Mimesis von Musik). ${ }^{3}$ Aufgrund der Differenzierung zwischen unterschiedlichen Codes der Schriftlichkeit und der Musik baut Schmidt schließlich eine eigene Methodologie zur Untersuchung spätromantischer intermedialer Musikästhetik. ${ }^{4}$ Schmidts Auffassung der Medienkombination dient als Grundlage für den vorliegenden Beitrag, wird aber weiterführend mit der Typologisierung der Intermedialität von Uwe Wirth ${ }^{5}$ und Evi Zemanek ${ }^{6}$ ergänzt.

Aufgrund der oben genannten theoretischen Ansätze, die unter anderem auf die Diskrepanzen in intermedialen Strukturen fokussieren, wird im vorliegenden Beitrag bei der vergleichenden Analyse der zwei Vertonungen des Gedichtes von Julius Bierbaum Traum durch die Dämmerung von Richard Strauss und Vítězslav Novák untersucht, wie unterschiedlich sich die oben beschriebenen intermedialen Konstellationen in diesen zwei Vertonungen realisieren. Schließlich soll aufgrund der vorgelegten Analyse Nováks spezifische Kompositionstechnik, die ich zum Zweck dieses Artikels „musikalische Pole-

1 SCHMIDT, Wolf Gerhard. Schriftlichkeit und Musikalisierung Prolegomena zu einer Theorie (spät)romantischer Medienkombination am Beispiel von Schumanns Eichendorff-Lied Mondnacht. Dtsch Vierteljahrsschr Literaturwiss Geistesgesch 79, 2005, S. 286-306.

2 Ebenda S. 287.

3 SHER, Paul. Literatur und Musik: Ein Handbuch zur Theorie und Praxis eines komparatistischen Grenzgebietes, Erich Schmidt Verlag, Berlin/Hannover 1984.

4 Vgl. SCHMIDT, Wolf Gerhard. Schriftlichkeit und Musikalisierung Prolegomena zu einer Theorie (spät) romantischer Medienkombination am Beispiel von Schumanns Eichendorff-Lied Mondnacht. Dtsch Vierteljahrsschr Literaturwiss Geistesgesch 79, 2005, S. 286-306. Hier S. 288-289.

5 WIRTH, Uwe. Hypertextualität als Gegenstand einer ,intermedialen Literaturwissenschaft in Walter Erhart (Hrsg.), Grenzen der Germanistik, Stuttgart/ Weimar: Metzler 2004, S. 410-430.

6 ZEMANEK, Evi. Intermedialität - Interart Studies, in Komparatistik, hg. v. Evi Zemanek u. Alexander Nebrig, Berlin 2012. 
mik“ nennen möchte und das sich direkt auf die intermedialen Bezüge bezieht, anschaulich gemacht werden.

\section{Modelle zur Erforschung von Intermedialitätsbezügen}

In der Einleitung habe ich darauf hingewiesen, dass in der Intermedialitätsforschung die Herangehensweise an intermediale Kunstwerke, die auf der Abgrenzbarkeit einzelner Medien baut, immer häufiger kritisiert wird. Neuere Ansätze der Intermedialitätsforschung von Zemanek, Schmidt oder Wirth betonen den Konstruktcharakter solcher Annahmen und beanstanden besonders die Parallelisierung des Begriffs der Intermedialität mit dem poststrukturalistischen Konzept der universalen Intertextualität, die schließlich dazu führen kann, dass alle Texte/Kunstwerke ebenso intermedial wie intertextuell betrachtet werden ${ }^{7}$. Zemanek schlägt deshalb vor, sich auf die Intensität der Intermedialität in verschiedenen Epochen sowie in verschiedenen Kunstwerken zu konzentrieren, die, wie Zemanek annimmt, deutlich variabel ist. Daher versucht sie, konkreten Untersuchungen einen dynamischen Intermedialitätsbegriff zugrunde zu legen, der die Unterscheidung verschiedener Arten und gradueller Abstufungen von Intermedialität zulässt. ${ }^{8}$ Dabei treten allerdings andere Schwierigkeiten ins Spiel, die bei der erweiterten Begriffsdefinition der einzelnen Intermedialitätsstufen $\mathrm{zu}$ begriffsdefinitorischen Unklarheiten führen können. So verlangt Zemanek, dass es sichergestellt werden muss, ob ,intermediale' Konfigurationen nicht mit ,transmedialen' (d. h. medienübergreifenden) Phänomenen verwechselt werden. ${ }^{9}$ Zemanek definiert die Unterscheidung zwischen diesen Phänomenen wie folgt:

„bei Ersteren beziehen sich verschiedene Medien aufeinander oder greifen ineinander; bei Letzteren bestehen medienunspezifische Gemeinsamkeiten, zum Beispiel in Themen- und Motivwahl, Stil oder Gestaltungsprinzip, ohne dass sich die Medien gegenseitig als solche thematisieren oder imitieren. " 10

Aus Zemaneks Abgrenzung ergibt sich unter anderem die Frage nach den Stufen der Interaktion zwischen den Medien, aber auch nach der Grenzüberschreitung im intermedialen Geflecht. Für den Begriff der Grenzüberschreitung im Zusammenhang mit der Intermedialitätstheorie plädiert auch der Literaturwissenschaftler Werner Wolf. Er betrachtet die Intermedialität als mediale Grenzüberschreitungen und Interferenzen im Bereich

7 Gilles Deleuze: „es gibt kein Kunstwerk, das nicht seine Fortsetzung oder seinen Ursprung in anderen Künsten hat" (Le cerveau, c’est l'écran. Entretien avec Gilles Deleuze“, Cahiers du Cinéma 380 (1986), 25-32, hier 28 (Übersetzung W.G.S.).

8 ZEMANEK, Evi. Intermedialität - Interart Studies. In Komparatistik, hg. v. Evi Zemanek u. Alexander Nebrig, Berlin 2012. S. 167.

9 Dasselbe verlangt auch RAJEWSKY, Irina O. Intermedialität, Tübingen / Basel 2002., S. $12 \mathrm{f}$.

10 ZEMANEK, Evi. 2012. S. 168. 
der Künste und der neuen Medien. ${ }^{11}$ Auch Rajewsky akzentuiert den Aspekt der Grenzüberschreitung, wenn sie vorschlägt, in der Diskussion der Mediengrenzen von „Ermöglichungsstrukturen“ auszugehen, womit das „dynamisch-performative Potential intermedialer Praktiken in den Vordergrund [rückt]“. ${ }^{12}$ In einfacher Form kann die Intermedialität daher dadurch charakterisiert werden, dass sie die Grenzen zwischen einzelnen Medien (Charakterkomplexe) überschreitet und einen Versuch darstellt, ein Zeichensystem in ein anderes zu integrieren.

Die Definition der Intermedialität aufgrund der Grenzüberschreitung zwischen den Medien ergänzt in seiner Typologie von unterschiedlichen Medienvernetzungen der Literaturwissenschaftler Uwe Wirth, der die Intermedialität genauso wie Zemanek oder Schmidt als ein dynamisches Konzept betrachtet und mehrere Stufen der Multi- und Intermedialität differenziert. Für Wirth ist Intermedialität als Grenzüberschreitung kein formales Konzept, ein Konzept, das einen Stil definiert, sondern berührt die Umsetzung eines breiteren Bereichs. Intermedialität geht über die üblichen Grenzen der Kunst hinaus, eröffnet einen neuen Raum und führt uns an die Grenzen der Kunst im Allgemeinen. Neben dem Begriff der Grenzüberschreitung führt Wirth aber auch den Begriff der Differenzqualität ein. Denn erst mit dem Bemerken ihrer medialen Differenzqualität würden die Medien beobachtbar, da die mediale Differenzqualität als Rahmungshinweis für die Grenze zwischen zwei Medien fungiert. ${ }^{13}$ Für die Beschreibung von intermedialen Bezügen ist demnach nicht nur das Phänomen der Grenzüberschreitung, sondern auch der Grenzmarkierung wichtig, indem die Grenzen der einzelnen Medien im intermedialen Bezug auffällig werden. Obwohl Wirth seine Typologie als vorläufig bezeichnet, wurde sie inzwischen in mehreren analytischen Arbeiten übernommen ${ }^{14}$, denn sie erlaubt das Phänomen der Intermedialitätsstufen in ihren Nuancen zu erfassen und deshalb möchte ich sie hier kurz darstellen, bevor ich sie als Ausgangspunkt für meine Analyse der „musikalischen Polemik“ bei Vítězslav Novák verwende.

Als Abgrenzung von der Intermedialität definiert Wirth zuerst die Multimedialität, also das Nebeneinander von Medien, die sich nach seiner Meinung von intermedialen Bezügen dadurch unterscheidet, dass es zu keinerlei Symbiose oder Konfrontation zwischen zwei Medien kommt. Die auf diese Weise aufgefasste Multimedialität entspricht als einzige den oben kritisierten Auffassungen der Intermedialität, die auf der Abgrenzbarkeit einzelner Medien bauen.

11 WERNER, Wolf. Intermedialität als neues Paradigma der Literaturwissenschaft?: Plädoyer für eine literaturzentrierte Erforschung von Grenzüberschreitungen zwischen Wortkunst und anderen Medien am Beispiel von Virginia Woolfs „The String Quartet“, in Arbeiten aus Anglistik und Amerikanistik, Vol. 21, No. 1 (1996), Narr Francke Attempto Verlag GmbH Co. KGS. 85-116. S. 86.

12 RAJEWSKY, Irina O. Intermediality, Intertextuality, and Remediation: A Literary Perspective on Intermediality, in Intermédialités, Nr. 6 (Montreal: University of Montreal), 2010, S. 43-64, hier. S. 47.

13 vgl. WIRTH, Uwe. Hypertextualität als Gegenstand einer, intermedialen Literaturwissenschaft, in Walter Erhart (Hrsg.), Grenzen der Germanistik, Stuttgart/ Weimar: Metzler 2004, S. 410-430. hier S. 417ff.

14 auswahlweise siehe auch KLEIN, David. Medienphantastik: Phantastische Literatur im Zeichen medialer Selbstreflexion bei Jorge Luis Borges und Julio Cortázar, Narr Dr. Gunter 2015., MAHLER, Andreas. Probleme der Intermedialitätsforschung: Medienbegriff - Interaktion - in Poetica, Brill 2012, S. 239-260. 
Als Nullstufe der Intermedialität betrachtet Wirth das Thematisieren eines Mediums in einem anderen Medium, etwa eine literarische Reflexion über die Malerei. Diese Stufe stellt lediglich mögliche Bedingungen für intermediale Bezüge, ohne dass sie tatsächlich umgesetzt werden.

Im Unterschied dazu wird die erste Stufe von Intermedialität nach Wirth als mediale Modulation der Konfiguration eines Zeichenverbundsystems dargestellt. Wirth spricht konkret über mediale Modulationen, in denen sich Übergänge gesprochener Sprache in geschriebene, von Handschrift zur Druckschrift und von Druckschrift in elektronische Schrift realisieren. Allerdings seien nicht alle medialen Modulationen als intermedial zu bezeichnen. Mediale Modulationen betrachtet Wirth erst dann als intermedial, „wenn sie zu einer Re-Konfiguration des Zeichenverbundsystems führen, wodurch sich dessen performative Verkörperungs- und Inszenierungsbedingungen ändern. "15

Die im vorliegenden Beitrag analysierten intermedialen Bezüge fallen unter die Stufe zwei. Diese definiert Wirth als eine Kopplung verschieden konfigurierter Zeichenverbundsysteme - etwa die Kopplung von Text und Bild. Wirth betont in diesem Zusammenhang den Unterschied zu einem multimedialen Nebeneinander, wobei das intermediale Miteinander durch eine integrierende konzeptionelle und mediale Re-Konfiguration ausgezeichnet. Eine derartige Re-Konfiguration impliziert eine technisch-mediale und eine inszenierend-konzeptionelle Modulation der performativen Rahmenbedingungen. Das Modell hierfür sind mediale Hybridbildung und mediale Aufpfropfung.

Die dritte Stufe der Intermedialität ist die konzeptionelle Aufpfropfung. Sie verzichtet auf eine mediale Modulation der Konfiguration bzw. auf die mediale Aufpfropfung verschieden konfigurierter Zeichenverbundsysteme. Stattdessen überträgt sie das Konzept der medialen Konfiguration eines Zeichenverbundsystems auf ein anderes. Hier könnte man neben der ut pictura poiesis und der poetischen Malerei die Übertragung theatraler Inszenierungsprinzipien auf die Buchgestaltung, die Idee einer konzeptionellen Mündlichkeit im Rahmen der Briefromanpoetik, den Einfluss der Schnitt-Technik des Films auf experimentelle Schreibweisen aber auch die Programmmusik oder bestimmte Formen der Konzeptkunst nennen. In all diesen Fällen wird das Konzept der Konfiguration eines Zeichenverbundsystems auf die mediale Konfiguration eines anderen Zeichenverbundsystems aufgepfropft.

Bei der Klassifizierung der Vertonung von Gedichten kommt es zu keiner metaphorischen Aufpropfung. Im Vertonungsprozess begegnen zwar sehr unterschiedlich konfigurierte Zeichensysteme einander, deren performative Rahmenbedingungen allerdings moduliert werden, wobei beide Zeichensysteme eine neue Qualität erhalten. Wirth spricht auch über die Intermedialität im engeren Sinne, oder harte Intermedialität. ${ }^{16}$

15 WIRTH, op. cit., 2004, hier S. 420.

16 WIRTH, op. cit., 2004, hier S. 421. 


\section{Intermediale Bezüge zwischen Literatur und Musik}

Die Frage inwieweit sich die zwei im vorliegenden Beitrag behandelten Zeichensysteme, also Musik und Sprache, unterscheiden, ist für eine intermediale Analyse der Gedichtsvertonungen besonders wichtig. Umberto Eco spricht über die Musik als über ein semiotisches System, das "rein syntaktisch und ohne offensichtliche semantische Dichte “ ist, ${ }^{17}$ Wolf Gerhard Schmidt hebt hervor, dass Musik - im Unterschied zur Sprache - „nichts bezeichnet, obwohl sie immer etwas bedeutet"18 und Carl Dahlhaus spricht über musikalische Logik, die zwischen Syntax und Semantik oszilliert. ${ }^{19}$ Nach Eggebrecht entsteht schließlich der Sinn in der Musik durch Bildung von Vokabeln, deren Bedeutungsgebung aber stark kontextabhängig ist. Anders als Sprache hat Musik kein wirkliches Denotat und ist in einem höheren Masse autoreflexiv als Literatur. ${ }^{20}$

Allerdings gibt es auch Gemeinsamkeiten zwischen der Musik und der Sprache, besonders zwischen der Musik und der Poesie. Musik und Poesie sind vergleichbar in rhetorischer Organisation ${ }^{21}$ und zeitorientierter Narrativität. ${ }^{22}$ Für Werner Wolf ist es der Ton, dessen Substanz sich bei der Verwendung in einer anderen Kunstsprache aus der intermedialen Perspektive nicht verändert und auf demselben Prinzip sowohl in der Musik als auch in der Poesie fungiert ${ }^{23}$. Peter V. Zima kommt zur Schlussfolgerung, dass das Wort als phonetische Substanz mit dem Ton der Musik verwandt ist. ${ }^{24}$ František Miko sieht den Unterschied zwischen Musik und Sprache in einer Hierarchisierung, in der die Sprache eine neuere semiotische Entwicklungsform der Musik ist. ${ }^{25}$ Für Otakar Zich verbindet die Poesiesprache selbst zwei selbstständige Funktionen: semantische und auditive. ${ }^{26}$ Zichs Beobachtung ist für die (spät)romantische intermediale Ästhetik, die im nächsten Kapitel untersucht wird, von Bedeutung. Die intermediale Verbindung

17 ECO Umberto. Einführung in die Semiotik, München 1972. S. $106 f$.

18 vgl. SCHMIDT, Wolf Gerhard: Schriftlichkeit und Musikalisierung Prolegomena zu einer Theorie (spät) romantischer Medienkombination am Beispiel von Schumanns Eichendorff-Lied Mondnacht. Dtsch Vierteljahrsschr Literaturwiss Geistesgesch 79, 2005, S. 286-306. hier S. 287.

19 DAHLHAUS, Carl. Die Idee der absoluten Musik, Kassel, Basel, Tours, London: Bärenreiter-Verlag - München : Deutscher Taschenbuch-Verlag 1978.

20 SCHMIDT, op. cit., 2005, hier S. 288., EGGEBRECHT, Hans Heinrich. Die Musik Gustav Mahlers, Wilhelmshaven : Noetzel 1999.

21 SCHANZE, Helmut. Die Gattung Lied im Spannungsfeld von Dichtung und Musik, in Wendt, Matthias (Hrsg.), Schumann und seine Dichter. Bericht über das 4 Internationale Schumann-Symposion am 13. und 14. Juni 1999 im Rahmen des 4. Schumann-Festes, Düsseldorf, Mainz 1993.

22 DANUSER, Hermann. Musik jenseits der Narrativität. Über Edgard Varèses Intégrales, in Helga de la Motte-Haber (Hrsg.), Edgard Varèse: Die Befreiung des Klangs. Symposium Edgard Varèse Hamburg 1991.

23 WOLF, Werner. The Muzicalization of Fiction. A Study in the Theory and History of Intermediality, Amsterdam: Rodopi, 1999.

24 ZIMA, Petr V. Literatur intermedial Musik - Malerei - Photographie - Film, Wissenschaftliche Buchgesellschaft 1995.

25 MIKO, František. Medzi rečou a hudbou, in derselbe Význam, jazyk, semióza, Nitra: Vysoká škola pedagogická, 1994, S. 95-104. hier S. 100.

26 ZICH, Otakar. O typech básnických, Orbis: Ars : sbírka rozprav o umění, Praha 1937. 
von Ton und Schrift wird in der (spät)romantischen Ästhetik nämlich als eine doppelte Musikalisierung aufgefasst, eine Vertonung des schon einmal im Gedicht vertonten Wortes wahr. ${ }^{27}$

\section{Medienkombinationen im Sinne (spät)romantischer Ästhetik}

Bei seiner Analyse des Liedes Mondnacht, einer Vertonung Schumanns auf das gleichnamige Gedicht von Eichendorff, versucht Wolf Gerhard Schmidt nachzuweisen, dass die intermediale Verbindung von Wort und Musik einen Ausgangspunkt der postromantischen Ästhetik darstellt. Aufgrund der spezifischen (spät)romantischen Kunstauffassung möchte er beweisen, dass gerade in der deutschen (spät)romantischen Liederkunst eine neue Differenz-Form-des-Dazwischen entsteht, wobei die oben dargestellten Differenzen zwischen Ton und Sprache durch eine neue intermediale Verbindung infrage gesetzt werden. Aus seiner Analyse von Mondnacht ergibt sich, dass es in diesem Falle um keine Modifikation der "Stimmung“ handelt, sonders es gehe um intermedial bedingte Bedeutungsverschiebung, dank der eine neue Form entsteht, in der der Ton durch das Wort und das Wort durch den Ton ineinander komplexer verflochten sind. Schmidt definiert diese Verflechtung als „,Musik'-Komplex, in dem jede der beiden Kunstformen Komponenten enthält, die - zumindest partiell auch der anderen Form zugehören " ${ }^{28}$ Er spricht über den Ort zwischen beiden Kunstformen. Das Verbindungelement, das dieser neuen Dimension entstehen lässt, ist die Singstimme, die Textlinie mit der Melodieführung verbindet. ${ }^{29}$ Somit kommt es zur Verflechtung von Ton und Sprache auf zwei unterschiedlichen Ebenen, erstens in der intermedialen Interferenz, wie oben beschrieben wurde. Zweitens aber auf der strukturellen Ebene. Dabei ist darauf zu fokussieren, welche Interferenzen zwischen der harmonischen Vertikale (Klavierpart) und der melodischen (in der (spät) romantischen Ästhetik zweifach musikalisierten) Horizontale (Wortmusik und Liedgesang) entstehen. Erst aus dieser zweifachen Vernetzung ergibt sich eine Raumstruktur, „die zugleich auf Defizientes wie Pleonastisches verweist und damit eine letztlich nie einholbare Totalität, umschreibt ‘ "“30

Was bedeutet allerdings genau Schmidts Behauptung, dass die melodische Horizontale zweifach musikalisiert ist? Auch diese Auffassung der Gedichtsvertonungen ist für die romantische Ästhetik geradezu spezifisch, denn sie ergibt sich aus dem allgemeinen romantischen Kunstverständnis. In der romantischen Weltanschauung erklingt die Welt nicht durch bloße Addition der Künste, sondern als Folge naturmagischer Inspiration. Jede Kunst ist somit ein Versuch, die „eigentümliche Grundmelodie“ der Natur auszudrücken, wobei dies nicht an ein bestimmtes Medium gebunden ist. Schon selbst die

27 SCHMIDT, op. cit., 2005, hier S. 296.

28 SCHMIDT, op. cit., 2005, hier S.305.

29 Vgl. ebenda, hier S. 296.

30 Ebenda, hier S. 305. 
Literatur, und besonders die Poesie, versucht die (absolute) Melodie der Natur nachzuahmen. Folglich wird im Sinne der (spät)romantischen Ästhetik die Vertonung literarischer Texte geradezu zu einer Tautologie, denn schon die Sprache selbst wirkt als Musik in Worten intermedial. ${ }^{31}$ Gabriele Leuenberger spricht von einer „musikalischen Formel“, welche sich in Eichendorffs Werken manifestiert: „Die Formeln sind aber musikalisch, weil ihr sprachmusikalisches Moment über das inhaltlich-begriffliche dominiert." 32 Sie bilden das Fundament, auf dem die einheitliche Textgenese aufbaut. Die musikalische Formel der romantischen Poesie ist dabei ein primär strukturell orientiertes Phänomen: der lautmalerische Gehalt der Worte überwiegt deren Substanz und Bedeutung. Gleichzeitig schafft der sprachmusikalische Gestus jedoch eine spezifische Semantik, in der sich „das ideele Anliegen des Dichters folglich als arschaisches Konzept der Weltharmonie mit religiöser Konnotation manifestiert." ${ }^{33}$ Leuenberger stellt in ihrer Analyse von Eichendorffs Gedichten fest, dass „die romantische Musikanschauung und -ästhetik von dem poetischen Ideal abgeleitet worden ist, um dann ihrerseits wieder idealiter in die Dichtkunst integriert zu werden." 34

Ich möchte im nachfolgenden analytischen Teil zeigen, dass erst diese von Schmidt, Leuenberger und anderen beschriebene (spät)romantische intermediale Ästhetik, in der die Musiksprache um eine neue Qualität erweitert wird, eine musikalische Polemik ermöglicht, so wie sie exemplarisch Vítězslav Novák in mehreren seiner Werke umgesetzt hat. Um diese neue Qualität beschreiben zu können, müssen wir uns auf die Problematik des Denotats in diesen zwei unterschiedlichen Systemen - dichterische Sprache und Tonsprache - konzentrieren. Im Rahmen der musikalischen Polemik, so wie sie von Novák realisiert wird, wird die Musikalisierung von Sprache als ein völlig neues Werk, eine Differenz-Form-des-Dazwischen aufgefasst, die ihre artifizielle Eigenständigkeit behält, aber zugleich in der intermedialen Realisierung die semantische Diskrepanz zwischen Wort und Musik verwischt und somit die ZuhörerInnen vor völlig neue Interpretationsdilemmata stellt. Mit jeder konkreten Realisierung einer Gedichtsvertonung kann somit mit unterschiedlichen komplexen Mitteln polemisiert werden. Dies umfasst nicht nur Zitate und Themenvariationen, wie dies bei anderen Autoren üblich war, sondern es handelt sich um eine komplexe polemisierende „Antwort“. Die bei diesem Prozess entstandenen Kompositionen können sicherlich als eigenständige Kunstwerke fungieren, dabei wird allerdings ihre intertextuelle und intermusikalische Dimension ausgeblendet. Um diese Werke komplex verstehen zu können, müssen sie sowohl durch das Prisma der intermedialen Ästhetik, wie sie in den (spät)romantischen Vertonungen realisiert wird, als auch als Reaktionen auf das Originalwerk betrachtet werden. Dies soll im Folgenden am

31 MOTTE-HABER, Helga de la. „Es flüstern und sprechen die Blumen...“ Zum Widerspruch zwischen Lied als romantischer Kategorie und musikalischer Gattung in Zeitschrift für Literaturwissenschaft und Linguistik; Göttingen Bd. 9, Ausg. 34, 70, 1979.

32 LEUENBERGER, Gabriele. Musikalischer Gestus und romantische Ästhetik : eine werkimmanente Poetik der Prosa Joseph von Eichendorffs, in Wilhelm Gössmann, Christoph Hollender (Hrsg.), Joseph von Eichendorff. Seine literarische und kulturelle Bedeutung, 2001, S. 137-138.

33 LEUENBERGER, op. cit., 2001, S. 139f.

34 Ebenda S. 95. 
Beispiel der Vertonungen Traum durch die Dämmerung von Richard Strauss und Vítězslav Novák gezeigt werden.

\section{Vítězslav Nováks „Musikaliche Polemik”}

Bei der Anwendung der oben präsentierten ästhetischen Kriterien wird im Nachfolgenden untersucht, worin eine komplexe, (spät)romantisch intermediale musikalische Polemik besteht. Dank den oben genannten Aspekten der postromantischen Ästhetik, wie sie von Schmidt und Leuenberger erkannt wurden, können verschiedene „polemisierende“ Kategorien identifiziert werden. Zusammenfassend handelt es sich um die Kategorien i) der Horizontale (Melodie) als auch ii) der harmonischen Vertikale, iii) der Interferenzen zwischen diesen und strukturelle Charakteristik, aber auch iv) der Auseinandersetzung mit dem Originaltext, seiner Fragmentierung, der Modulationen der originellen Phrasierung u.a. Aufgrund dieser vier Kategorien möchte ich zeigen, worin eine komplexe musikalische Polemik bei Novák besteht und inwieweit sie der (spät)romantischen Ästhetik entspricht. Zuerst werde ich die zwei Vertonungen von Novák und Strauss kurz vorstellen und nachfolgend vergleichen.

Der Autor des Gedichts Traum durch die Dämmerung ist Otto Julius Bierbaum ${ }^{35}$, der es im Jahre 1901 in seinem Sammelband von Gedichten aus den Jahren 1895-1900 mit dem Titel Irrgarten der Liebe veröffentlichte. Doch von Strauss wurde das Gedicht schon im Jahre seiner Entstehung, also 1895 zusammen mit zwei anderen Gedichten im Zyklus Drei Lieder (op. 29, 1895) vertont. Strauss vertonte auch weitere Texte von Bierbaum, zum Beispiel Freundliche Vision (Fünf Lieder nach Gedichten von Otto Julius Bierbaum und Karl Henckell, op. 48, 1898) und Junghexenlied (Fünf Lieder, op. 39, 1898). Vítězslav Nováks polemische Reaktion auf Strauss Vertonung entstand im Jahre 1912 als Teil der 6 Lieder auf Gedichte deutschsprachiger Autoren (op. 46). Sie war nicht die erste polemisierende Komposition Nováks - auf gleiche Weise polemisierte er mit Emanuel Chvála (Slovácká suita), Josef Richard Rozkošný und Josef Bohuslav Foerster (Šest mužských sborů op. 37, 1906), Zdeněk Fibich (Toman a lesní panna), František Neumann (Bouře) und ebenfalls mit Antonín Dvořák (Svatební košile). ${ }^{36}$ Nováks musikalische Polemik ist nicht nur eine andere Version des Originals wie Sýkora behauptet, sondern, wie im vorliegenden Beitrag gezeigt wird, eine komplexe, intermediale, auf mehreren Ebenen aufgebaute polemische Antwort. Im Folgenden werde ich auf die vier oben vorgestellten (spät)romantisch ästhetischen Kriterien detaillierter eingehen.

35 Zu Bierbaum siehe Otto Julius Bierbaum - Akteur im Netzwerk der Moderne [Björn Weyand / Bernd Zegowitz (Hg.).

36 SÝKORA, Pavel. Vítězslav Novák [online]. [cit. 2021-05-05]. Český hudebni slovnik osob a institucí. URL: https://www.ceskyhudebnislovnik.cz/slovnik/index.php?option=com_mdictionary\&task=. 


\section{Polemisierende Melodieführung}

Als erstes möchte ich mich mit der Melodieführung befassen, die für die (post)romantishce Ästhetik geradezu entscheidend ist. Schaut man sich jeweils den Anfang der Vertonungen von Strauss und Novák, stellt man fest, dass Novák Strauss Melodieführung spiegelartig negiert, wobei er die rhythmische Versgestaltung der Phrase übernimmt (Siehe Abb. 1 und 2).

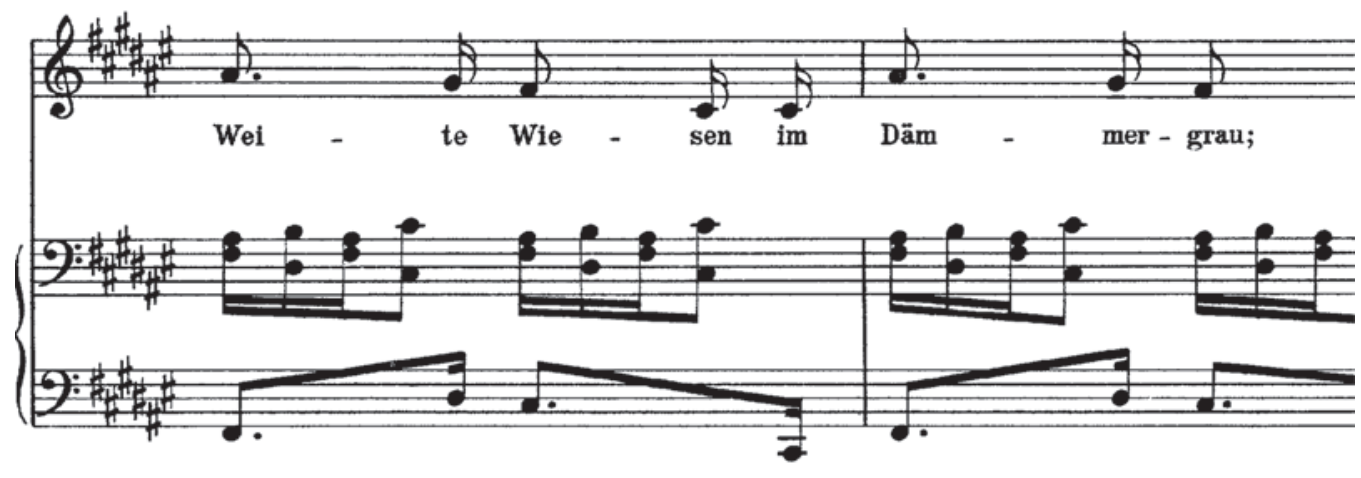

Abb. 1 Strauss' Melodieführung (Anfang).

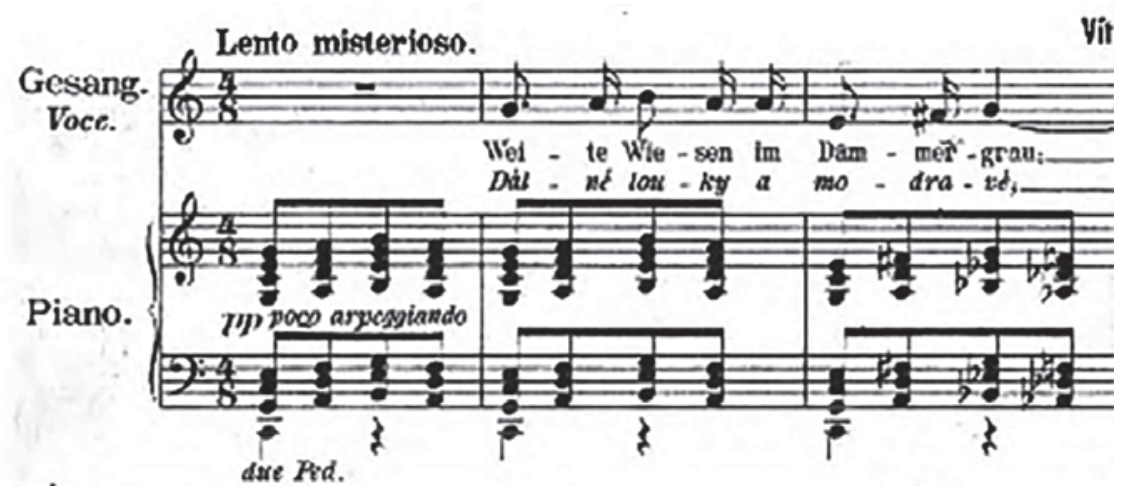

Abb. 2 Nováks Melodieführung (Anfang).

Die Modulation besteht aber auch in der Verschiebung von Fis Dur bei Strauss ins C Dur bei Novák, wobei allerdings die Melodieführung bei Novák eigentlich in e moll erklingt. Doch gleich im zweiten Vers moduliert Strauss ins B dur, Novák reagiert auf diese Änderung mit einer Es-dur-Modulation. Diese Modulationen gehören eigentlich zur musikalischen Vertikale, doch sie werden von der Melodieführung (die Horizontale) in diesem Fall gerade explizit eingeführt (siehe Abb. 3. und 4). 


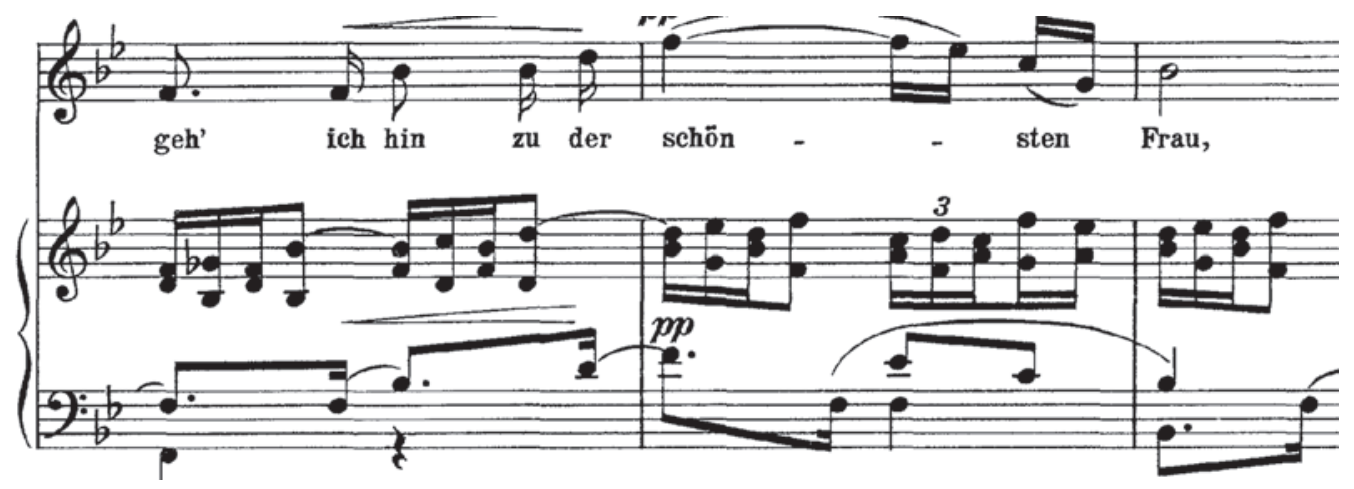

Abb. 3 Strauss, Takt Nr. 7-9.

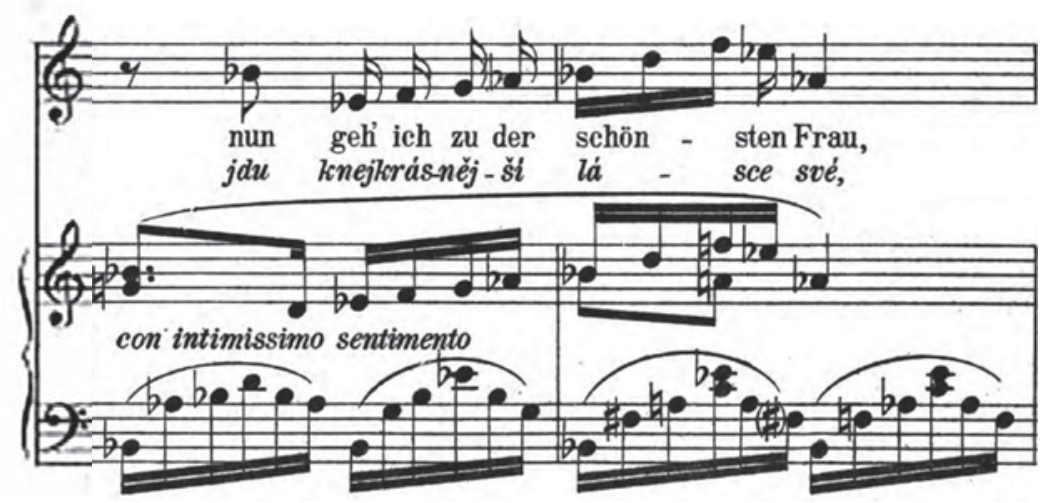

Abb 4. Novák, Takt Nr. 7-8.

Während Strauss die Phrasierung des ersten Verses im zweiten Vers wiederholt und somit die Musik vor der sprachlichen Versgestaltung bevorzugt, ist für die weitere Melodieführung bei Novák das Originalgedicht (die Sprache) entscheidend. Statt eines langen hohen Tons auf „schönen Frau“ bietet Novák einen emotionellen Lauf, der mit der ersten Hälfte des zweiten Verses („nun geh ich zu der“) einen musikalischen Bogen bildet. In Nováks Version lässt sich somit eine intermediale Symbiose von Musik und Sprache beobachten, wobei es bei Strauss zu einem intermedialen Konflikt kommt, in dem die musikalische gegenüber der sprachlichen Gestaltung bevorzugt wird.

Am Ende der ersten Strophe entspricht Strauss' Melodieführung der Form des Gedichts und sinkt zum des ${ }^{1}$, womit eine klare Zäsur zwischen der ersten und der zweiten Strophe entsteht (siehe Abb. 5). 


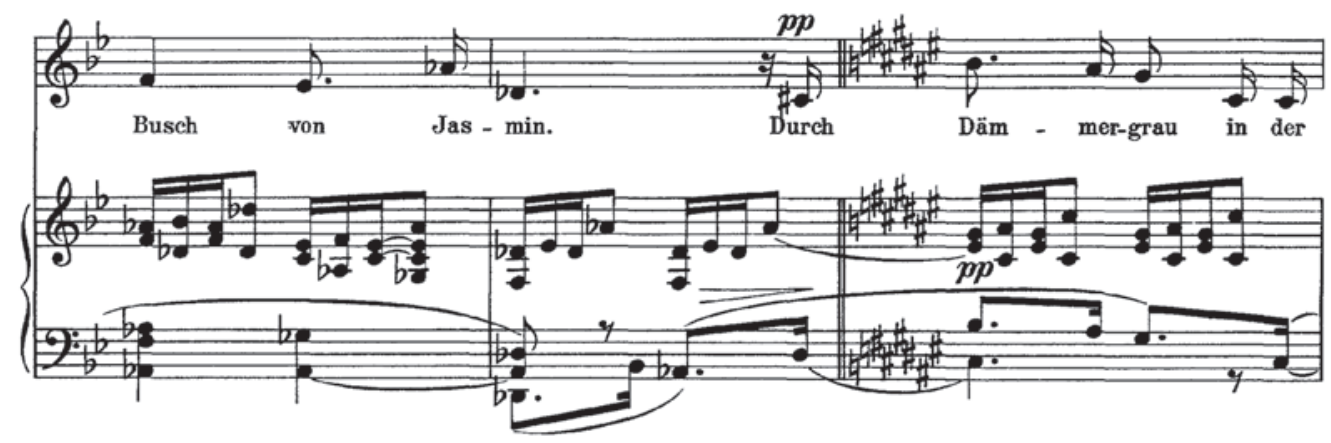

Abb. 5 Strauss, Takt Nr. 13-15, Ende der ersten Strophe.

Demgegenüber steigt bei Novák die Melodieführung zum $\mathrm{g}^{1} \mathrm{zu}$ einem offenen Ende der ersten Strophe, die Zäsur zwischen der ersten und zweiten Strophe realisiert sich somit nicht in der melodischen Horizontale, sondern in der Begleitung im kurzen Zwischenspiel, in dem die rhythmische sowie harmonische Satzung moduliert werden.
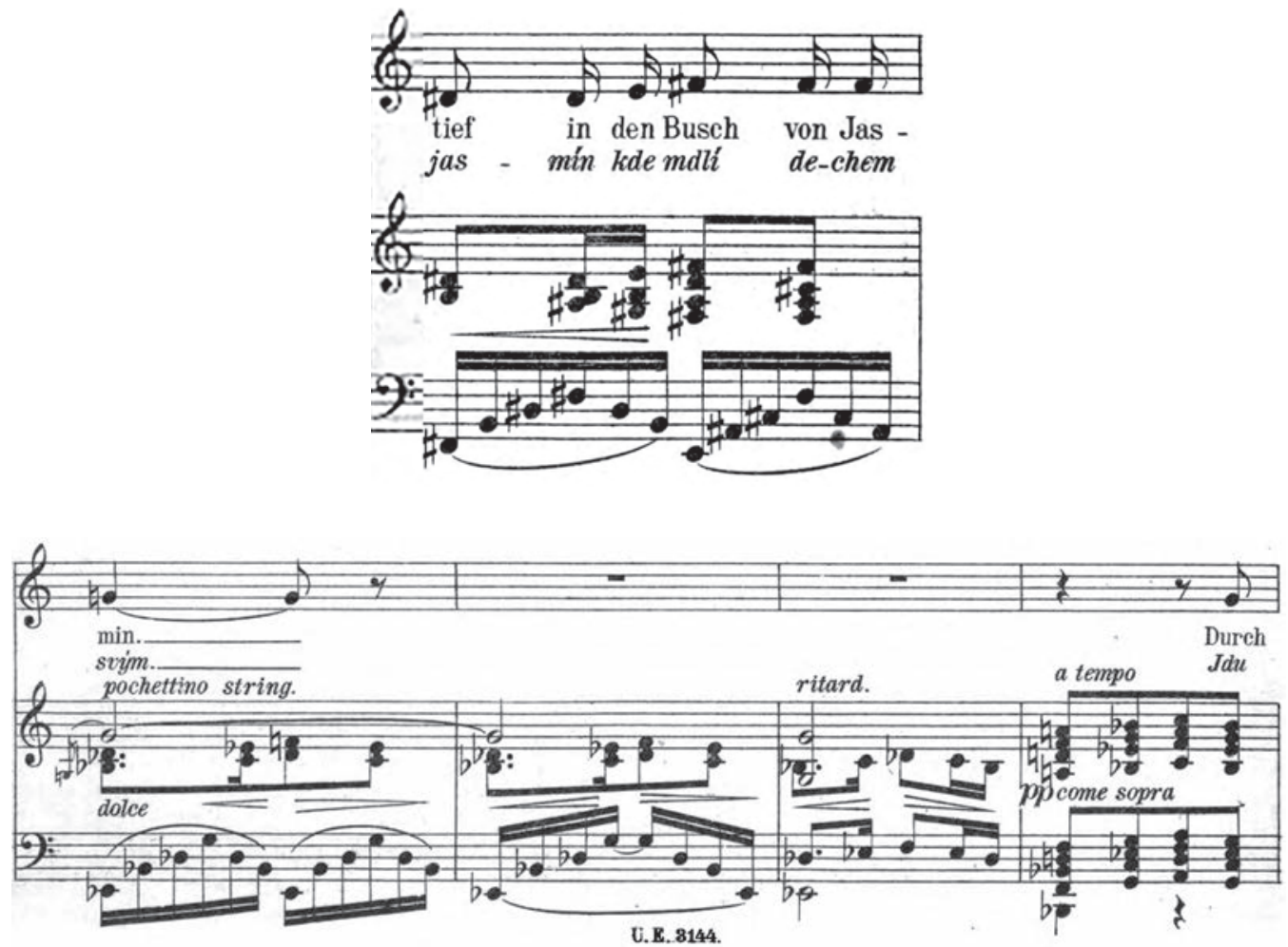

Abb. 6 Novák, Takt Nr. 11-15, Ende der ersten Strophe. 
Vergleicht man das Ende der ersten Strophe mit dem Ende des Liedes, wird klar, inwieweit Strauss' Melodieführung bei Novák polemisierend umgestaltet wird. Zum Schluss des Liedes lässt Strauss die Melodie in ein offenes Ende bis auf c ${ }^{2}$ aufsteigen (siehe Abb. 7), während Novák die Melodie sinkend führt und auf es ${ }^{1}$ abschließt (siehe Abb. 8). Daraus lässt sich ableiten, dass Nováks Version auch auf der Makroebene eine absichtlich genaue Negation der Vertonung von Strauss darstellt.

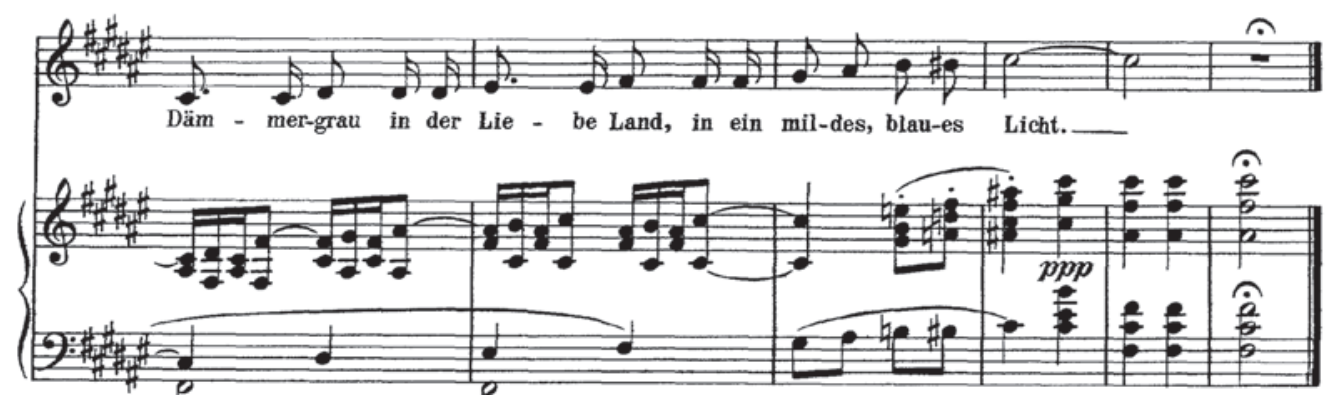

Abb. 7 Strauss Takt Nr. 28-33, Ende der zweiten Strophe.

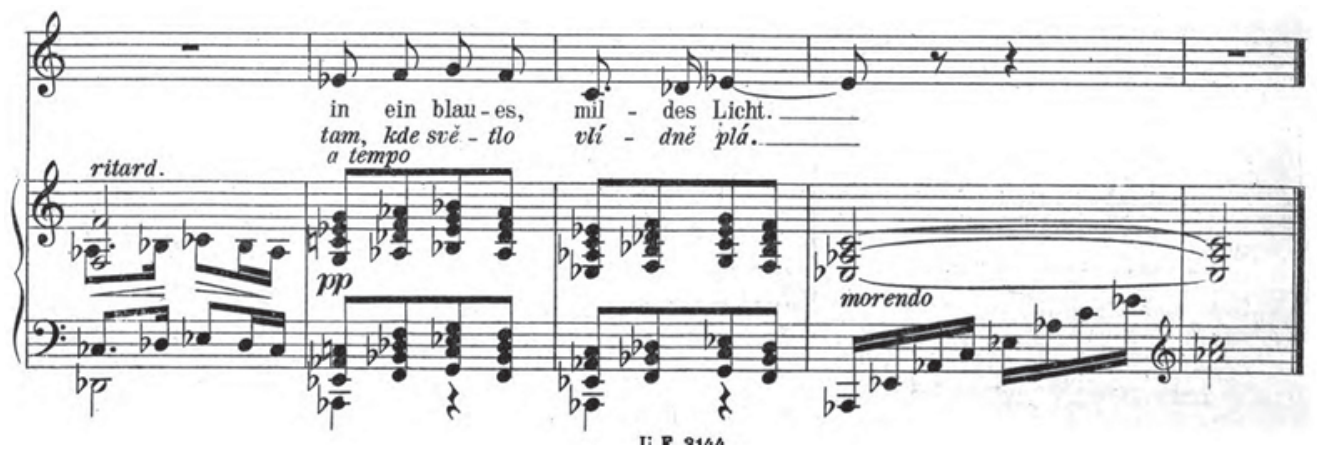

Abb. 8 Novák, Takt Nr. 28-32, Ende der zweiten Strophe.

An den oben genannten Beispielen wird anschaulich gezeigt, dass Nováks Polemik geradezu kontradiktorische Version in der Melodieführung (spiegelartige Variationen, Bevorzugung der Sprache gegenüber der Musikalisierung, umgekehrte Gestaltung der letzten Verse der Strophen und am Schluss des Liedes) bietet.

\section{Polemisierende Harmonisierung}

Aus der vergleichenden Analyse der Melodieführung im vorangegangenen Absatz ergaben sich unter anderem auch Unterschiede in der vertikalen Gestaltung der Vertonungen 
von Novák und Strauss. Nováks Version oszilliert zwischen Dur-moll Tonarten und eröffnet sich am Ende in eine neue unerwartete Tonart (As Dur), während Strauss in die einleitende Fis-Dur-Tonart über Modulationen zurückkehrt (vergleiche Abb. 1, 2 und 7, 8). Novák polemisiert mit Strauss' Vertonung auch in der Modulation zwischen beiden Strophen. Während Strauss vom Des Dur ins Gis Dur moduliert und somit gegenüber dem einleitenden Fis Dur die gleiche Melodie in der zweiten Strophe um einen Ton höher wiederholt, leitet Novák die erste Strophe im C Dur ein, entwickelt die Melodie spiegelartig zu Strauss' Melodieführung von $\mathrm{g}^{1}$ in der Dur-moll-Oszillation und schließlich moduliert er in der zweiten Strophe um eine Quarte höher ins F Dur, die Melodie sinkt allerdings um einen Ton tiefer auf $\mathrm{F}$ und die ursprüngliche Dur-moll-Spannung aus der ersten Strophe wird in der zweiten Strophe aufgehoben (siehe Abb. 6). Im Takt Nr. 28 moduliert Novák um einen Ton tiefer ins Es Dur/As Dur, wobei das gleiche melodische Motiv der harmonischen Modulation entsprechend sinkt, um das Klavier im hohen As Dur abschließen zu lassen (siehe Abb. 8).

Mit der Klaviersatzung polemisiert Novák deutlich schon am Anfang des Liedes (siehe Abb. 9 und 10), in dem er die linke und rechte Hand rhythmisch parallel spielen lässt, wobei Strauss gleich im ersten Takt die für seine Vertonung prägende Kontrastwirkung der Triolen gegenüber den punktieren Achteln entstehen lässt.

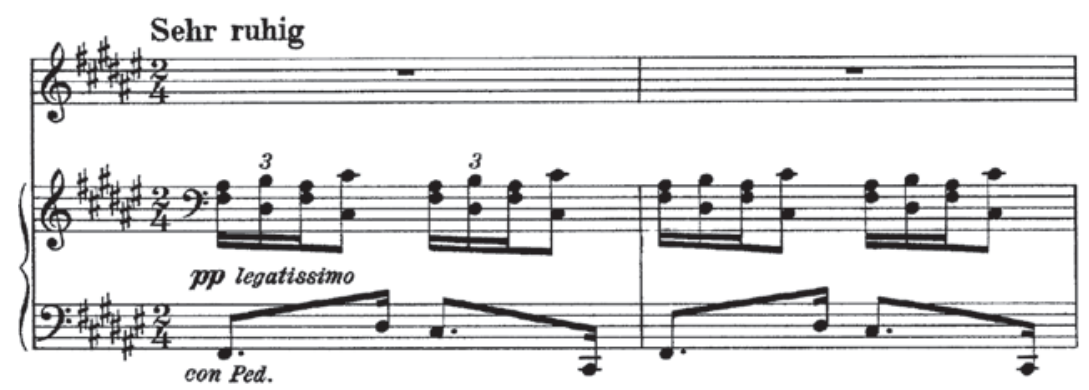

Abb. 9 Strauss, Takt 1, Anfang.

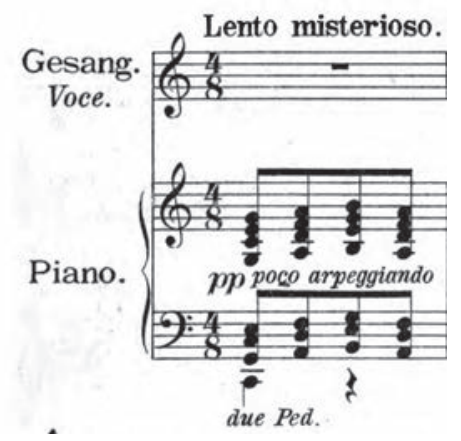

Abb. 10 Novák, Takt 1, Anfang. 
Oben konnte nachgewiesen werden, dass Novák auch in der harmonischen Satzung und im Klavierpart (Vertikale) mit Strauss ' Vertonung polemisiert und die Klavierbegleitung als Antwort auf verschiedenen Ebenen gestaltet - a) in der Dur-moll-Modulation, b) in der Modulation zwischen der ersten und der zweiten Strophe und c) am Schluss des Liedes mit der Modulation in eine ganz neue unerwartete Tonart.

\section{Interferenzen zwischen der Horizontale und der Vertikale}

Auch in der Vernetzung der melodischen Horizontale mit der harmonischen Vertikale finden wir in Nováks Version eindeutige polemisierende Elemente, die als Antwort auf Strauss' Original aufzufassen sind. Wie oben dargeboten wurde, baut Strauss auf dem Kontrast zwischen den Triolen in der rechten Hand im Klavier und der Punktierung in der linken Hand im Klavier und in der Stimme (siehe Abb 1.), während bei Novák das Klavier in Vierteln mit der Melodie rhythmisch parallel spielt (siehe Abb 2), um im zweiten Vers in Sechzehntel-Triolen zu übergehen und somit einen Kontrast zu der Melodieführung in einfachen Sechszehnteln zu entwickeln (siehe Abb. 4). Strauss bietet der Singstimme fast immer eine Unterstützung im Klavier und verstärkt somit den Kontrast zwischen den Triolen und den (punktierten) Achteln in der linken Hand - anders als in anderen hier erwähnten Aspekten polemisiert Novák mit diesem Schema überraschenderweise nicht, ganz im Gegenteil, er übernimmt es (siehe Abb. 6, Takt Nr. 13-15). Auch hier realisiert er allerdings eine spiegelartige Variation, denn während bei Strauss die Triolen in der rechten und die punktierten Achtel in der linken Hand gespielt werden, ist es bei Novák genau umgekehrt.

\section{Polemisierende Auffassung der Rollen von Sprache und Musik}

An allen oben genannten Kategorien konnte nachgewiesen werden, dass Nováks Polemik als eine komplexe Variation aufzufassen ist. Neben dem Aufbau, der Melodieführung der harmonischen Satzung und deren Interferenzen ist es aber auch der rein intermediale Aspekt, der im Vertonungsprozess geradezu eine Schlüsselrolle spielt. Die medialen Grenzüberschreitungen und Interferenzen zwischen den Medien treten bei der vorliegenden vergleichenden Analyse von Nováks und Strauss 'Vertonungen von Traum durch die Dämmerung deutlich hervor. Strauss befolgt im Vertonungsprozess streng formalistisch den Aufbau des Gedichts - beide Strophen folgen derselben Phrasierung, Verse werden entsprechend eingehalten, lediglich am Ende wird durch die Wiederholung des letzten Verses das Original den Anforderungen der musikalischen Form angepasst, die Mikrostruktur - die sprachliche Gestaltung des Originals ist für Strauss offensichtlich weniger wichtig als die musikalischen Aspekte. Seine Vertonung ist folglich klar in zwei Bögen strukturiert und im Vergleich mit Novák auch relativ monoton. Novák hält sich 
zwar auch an der durch das Original festgelegten Struktur, übernimmt allerdings bei der Melodieführung die Phrasierung der Sprache - er fragmentiert die Melodie und unterstützt die emotionellen Phrasen mit dynamischer Melodie- und Begleitführung (siehe Abb. 4 und 11), seine Vertonung ist aufgrund dieser bewusst intermedialen Symbiose zwischen Sprache und Musik dynamischer und expressiver.

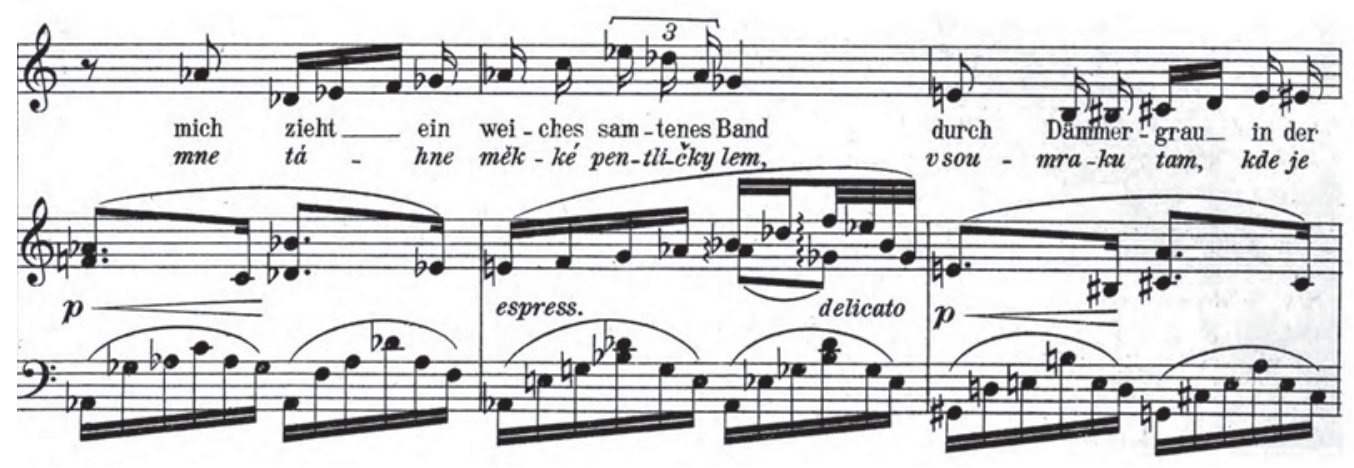

Abb 11. Novák, Takt Nr. 21-23.

\section{Fazit}

Im vorliegenden Beitrag wird Vítězslav Nováks Vorgehen der musikalischen Polemik mithilfe von Konzepten der intermedialen (spät)romantischen Ästhetik dargestellt. Es zeigt sich, dass erst die (spät)romantische komplexe Auffassung der Intermedialität, wie sie von Schmidt und Leuenberger beschrieben wurde, eine grundlegende Analyse von diesem in der Musikgeschichte unüblichen Kompositionsverfahren ermöglicht. Wichtig für eine solche Analyse sind Definitionen der Intermedialität als eines Phänomens, bei dem die Grenzüberschreitung und zugleich die Qualitätsdifferenz und Grenzmarkierung von verschiedenen Medien in den Fokus rücken. Erst bei der vergleichenden Analyse der Vertonungen von Strauss und Novák auf den Ebenen i) der musikalischen Horizontale (Melodieführung), ii) der musikalischen Vertikale (harmonische Gestaltung, Begleitung), iii) der Interferenzen zwischen der Horizontale und der Vertikale, iv) der Funktion der Sprache und der Musik im Kompositionsprozess tritt die Komplexität der musikalischen Polemik in Nováks Kompositionen ans Licht. Es zeigt sich dabei, dass es sich bei Novák nicht nur um eine Antwort, beziehungsweise eine andere Version, oder bloße Variation des Originals handelt, wie dies beispielsweise in Český hudební slovník erwähnt wird, sondern es geht vielmehr um eine komplexe auf mehreren Ebenen polemisierende Bearbeitung. Betrachten wir diese Feststellung im Prisma der allgemeinen Auffassung der (spät)romantischen Ästhetik, scheint es, dass auch die verbreitete, omnipräsente These, dass diese 
die absolute Musik favorisiere, ${ }^{37} \mathrm{zu}$ revidieren wäre. Denn, wie sich aus der vorliegenden Analyse ergibt, intermediale Bezüge zwischen Musik und Sprache realisieren sich in der (spät)romantischen Ästhetik auf unterschiedlichen Ebenen in unterschiedlicher Qualität, weshalb zumindest im Falle von (spät)romantischen Gedichtsvertonungen nicht über absolute Musik gesprochen werden kann, sondern über eine Differenzform-des-Dazwischen, ${ }^{38}$ eine Form, die gegenüber der Idee der absoluten Musik die intermediale Vernetzung von Musik und Sprache hervorhebt.

\section{Bibliography}

\section{Primärquellen:}

NOVÁK Vítězslav. Traum durch die Dämmerung in Erotikon, Verlag Universal Edition (1912).

STRAUSS, Richard. Traum durch die Dämmerung in Drei Lieder, Verlag Universal Edition.

\section{Literaturverzeichnis:}

DAHLHAUS, Carl. Die Idee der absoluten Musik, Kassel, Basel, Tours, London : Bärenreiter-Verlag - München : Deutscher Taschenbuch-Verlag 1978.

DANUSER, Hermann. Musik jenseits der Narrativität. Über Edgard Varèses Intégrales, in Helga de la Motte-Haber (Hrsg.), Edgard Varèse: Die Befreiung des Klangs. Symposium Edgard Varèse Hamburg 1991.

ECO, Umberto. Einführung in die Semiotik, Fink, München 1972.

EGGEBRECHT, Hans Heinrich. Die Musik Gustav Mahlers, Wilhelmshaven : Noetzel 1999.

KLEIN, David. Medienphantastik: Phantastische Literatur im Zeichen medialer Selbstreflexion bei Jorge Luis Borges und Julio Cortázar, Narr Dr. Gunter 2015.

LEUENBERGER, Gabriele. Musikalischer Gestus und romantische Ästhetik : eine werkimmanente Poetik der Prosa Joseph von Eichendorffs, in: Wilhelm Gössmann, Christoph Hollender (Hrsg.), Joseph von Eichendorff. Seine literarische und kulturelle Bedeutung Paderborn [u.a.] : Schöningh 1995, S. 79-141.

MAHLER, Andreas. Probleme der Intermedialitätsforschung: Medienbegriff - Interaktion - in Poetica, Brill 2012, S. 239-260.

MIKO, František. Medzi rečou a hudbou, in derselbe Význam, jazyk, semióza, Nitra: Vysoká škola pedagogická, 1994, S. 95-104.

MOTTE-HABER, Helga de la. „Es flüstern und sprechen die Blumen... “ Zum Widerspruch zwischen Lied als romantischer Kategorie und musikalischer Gattung in Zeitschrift für Literaturwissenschaft und Linguistik; Göttingen Bd. 9, Ausg. 34, 70, 1979.

37 DAHLHAUS 1978.

38 SCHMIDT 2005, S. 286-306. 
RAJEWSKY, Irina O. Intermediality, Intertextuality, and Remediation: A Literary Perspective on Intermediality, in Intermédialités, Nr. 6 (Montreal: University of Montreal), 2005, S. 43-64.

RAJEWSKY, Irina O. Intermedialität, Tübingen / Basel 2002.

SCHANZE, Helmut. Die Gattung Lied im Spannungsfeld von Dichtung und Musik, in: Wendt, Matthias (Hrsg.), Schumann und seine Dichter. Bericht über das 4 Internationale Schumann-Symposion am 13. und 14. Juni 1999 im Rahmen des 4. Schumann-Festes, Düsseldorf, Mainz 1993.

SCHMIDT, Wolf Gerhard. Schriftlichkeit und Musikalisierung Prolegomena zu einer Theorie (spät)romantischer Medienkombination am Beispiel von Schumanns Eichendorff-Lied Mondnacht. Dtsch Vierteljahrsschr Literaturwiss Geistesgesch 79, 2005, S. 286-306.

SHER, Paul. Literatur und Musik: Ein Handbuch zur Theorie und Praxis eines komparatistischen Grenzgebietes, Erich Schmidt Verlag, Berlin/Hannover 1984.

SÝKORA, Pavel. Vítězslav Novák [online]. [cit. 2021-05-05]. Český hudebni slovnik osob a instituci. URL: https://www.ceskyhudebnislovnik.cz/slovnik/index.php?option=com_mdictionary\&task $=$.

WEAND, Bjorn; ZEGOWITZ Bernd. Otto Julius Bierbaum - Akteur im Netzwerk der Moderne Quintus Verlag, Berlin 2018.

WIRTH, Uwe. Hypertextualität als Gegenstand einer intermedialen Literaturwissenschaft in Walter Erhart (Hrsg.), Grenzen der Germanistik, Stuttgart/ Weimar: Metzler 2004, S. 410-430.

WOLF, Werner. The Muzicalization of Fiction. A Study in the Theory and History of Intermediality, Amsterdam: Rodopi, 1999.

WOLF, Werner. Intermedialität als neues Paradigma der Literaturwissenschaft?: Plädoyer für eine literaturzentrierte Erforschung von Grenzüberschreitungen zwischen Wortkunst und anderen Medien am Beispiel von Virginia Woolfs „The String Quartet“, in: Arbeiten aus Anglistik und Amerikanistik, Vol. 21, No. 1 (1996), Narr Francke Attempto Verlag GmbH Co. KGS. 85-116

ZEMANEK, Evi. Intermedialität - Interart Studies. In: Komparatistik, hg. v. Evi Zemanek u. Alexander Nebrig, Berlin 2012.

ZICH, Otakar. O typech básnických, Orbis: Ars : sbírka rozprav o umění, Praha 1937.

ZIMA, Petr V. Literatur intermedial Musik - Malerei - Photographie - Film, Wissenschaftliche Buchgesellschaft 1995 . 